\title{
The obligate intracellular lifestyle
}

\author{
Kenneth A. Fields ${ }^{1}{ }^{*}$, Robert A. Heinzen ${ }^{2}$ and Rey Carabeo ${ }^{3}$ \\ 1 Department of Microbiology and Immunology, University of Miami Miller School of Medicine, Miami, FL, USA \\ 2 Coxiella Pathogenesis Section Rocky Mountain Laboratories, National Institute of Allergy and Infectious Diseases, National Institutes of Health, Hamilton, MT, USA \\ ${ }^{3}$ Division of Cell and Molecular Biology, Centre for Molecular Microbiology and Infection, Imperial College, London, UK \\ *Correspondence: kfields@med.miami.edu
}

Obligate intracellular bacteria represent consummate parasites, often covertly coopting host resources to enable development and ultimately transmission to a new host. The overall success of this survival strategy is doubtless derived from co-evolution with respective eukaryotic hosts over hundreds of millions of years. Indeed, many species of obligate intracellular bacteria represent pathogens capable of significant negative impact on worldwide human health. This link to human disease and the fascinating infection biology exhibited by these parasites render them exquisite subjects for investigation. Despite the overarching absolute requirement for growth within eukaryotic cells, this class of bacteria has evolved distinct strategies that enable colonization of diverse tissues, cell types, and even subcellular niches. We have assembled a collection of Opinion, Review, and Primary Research articles that delve into the often unique biology of obligate intracellular bacteria. The reader of this Special Topics Edition will find examples of virulence strategies employed by Chlamydia, Anaplasma, Ehrlichia, and Rickettsia. We have also included Coxiella in our consideration of obligate intracellular bacteria. Despite the recent development of a host cell-independent culture method (see the review by Beare et al., 2011), Coxiella burnetii remains confined to intracellular growth under natural settings. Aspects of covered infection biology include mechanisms of host cell invasion, production, and secretion of anti-host proteins, nutrient acquisition, and host immune response. Unfortunately, the biology that renders this class of microbes so interesting has also often thrown up barriers that complicate investigation. These issues are also touched upon in articles that will hopefully point the way forward.

The ability to invade and gain access to the host cell interior is of obvious importance to obligate intracellular bac- teria. However, the precise mechanisms for adherence and invasion remain unresolved for most species. Chan et al. (2010) reviews the current knowledge regarding adherence and invasion by Rickettsia spp. and highlights how interference with these events could lead to novel modes of prevention and treatment. This is particularly important since the efficacy of the host immune response can be limited in response to this class of parasites. While the obligate intracellular lifestyle certainly shields pathogens from host defense mechanisms somewhat, some parasite proteins are highly immunogenic. Gall et al. (2011) characterizes in vitro and in vivo immune responses to chlamydial antigens, and their report raises questions regarding how the immune response contributes to the pathology associated with chlamydial disease.

In contrast to Rickettsia spp., intracellular development of C. burnetii and Chlamydia spp. occurs within a membrane-bound parasitophorous vacuole. Such sequestration presents unique challenges for these organisms and necessitates mechanisms to establish and maintain this unique compartment. Hussain et al. (2011) investigated contributions of eukaryotic factors in formation of the C. burnetii-containing vacuole and present evidence that multiple host kinases are essential for vacuole biogenesis. This theme is further explored by Ouellette and Carabeo (2010)who describe an example of how obligate intracellular bacteria intimately interact with and depend on host resources. They demonstrate that recycling rates of transferring-containing vesicles are important for optimal chlamydial growth. Whether this pathway is required to deliver iron was unclear, yet a Methods report from Thompson and Carabeo (2011) illustrates the importance of iron in chlamydial development and establishes a novel method to induce iron starvation during infections with obligate intracellular bacteria.
Similar to the case with their facultative cousins, secretion of host-interactive effector proteins represents a significant mechanism employed by obligate intracellular bacteria to promote virulence through modulation of host cell processes. Therefore, no consideration of obligate intracellular microbiology would be complete without including current views of protein secretion and effector protein function. BettsHampikian and Fields (2010) provide a thorough review of the chlamydial type III secretion mechanism and emphasize findings that indicate unique adaptations to the obligate intracellular lifestyle. Stone et al. (2011) extends this discussion in an original research article exploring molecular mechanisms employed to regulate activity of the secretion apparatus. Finally, two articles explore specific effector protein function. Zhong (2011) broadly explores the role of secreted proteases in sculpting the intracellular host environment while simultaneously generating a pool of amino acids for use by parasite, while Broederdorf and Voth (2011) provides an interesting commentary regarding the anti-apoptotic mechanisms of a type IV-secreted C. burnetii effector protein.

Investigating the infection biology of obligate intracellular bacteria is often a complicated process. First, psychological barriers can exist that make researchers hesitate to even carry out research. For example, Wolf (2011) describes the dilemma currently limiting interest regarding the pathogenesis of Chlamydia pneumoniae. However, the lack of tractable genetic system represents perhaps the greatest barrier confounding significant progress in obligate intracellular biology. In the past, rigorous proteomic studies have helped drive progress in the absence of mutant generation. For example, the proteomic analyses of Anaplasma and Ehrlichia performed by Lin et al. (2011) illustrate how these studies can provide useful insight. Happily, the future now seems 
bright for some degree of genetic manipulation. Beare et al. (2011) provide a thoughtful review of factors that have confounded efforts in the past and summarize exciting advances where real progress has been made in genetic analyses of obligate intracellular bacteria. Significantly, advances have also come been made in historically intractable Chlamydia system where it is now possible to generate targeted mutations in chlamydial genes. As the sampling of articles in this Special Topics issue illustrates, the biology and pathogenesis of obligate intracellular bacteria represents a fertile area of investigation that is likely poised to expand rapidly in the future.

\section{REFERENCES}

Beare, P., A., Sandoz, K., M., Omsland, A., Rockey, D., D., and Heinzen, R. A. (2011). Advances in genetic manipulation of obligate intracellular bacteria pathogens. Front. Microbio. 2:97. doi: 10.3389/ fmicb.2011.00097

Betts-Hampikian, H., J. and Fields, K. A. (2010). The chlamydial type III secretion mechanism: revealing cracks in a tough nut. Front. Microbio. 1:114. doi: 10.3389/fmicb.2010.00114

Broederdorf, L., J. and Voth, D. E. (2011). Cheating Death: A Coxiella effector prevents apoptosis. Front. Microbio. 2:43. doi: 10.3389/fmicb.2011.00043

Chan, Y., G., Riley, S., P., and Martinez, J. J. (2010). Adherence to and invasion of host cells by spotted fever group Rickettsia. Front. Microbio. 1:139. doi: 10.3389/fmicb.2010.00139

Gall,A., Horowitz,A., Joof,H., Natividad,A., Tetteh, K., Riley, E., Bailey, R., L., Mabey, D., C., and Holland, M. J. (2011). Systemic effector and regulatory immune responses to chlamydial antigens in Trachomatous Trichiasis. Front. Microbio. 2:10. doi: 10.3389/fmicb.2011.00010

Hussain, S., K., Broederdorf, L., J., Sharma, U., M., and Voth, D. E. (2011). Host kinase activity is required for Coxiella burnetii parasitophorous vacuole formation. Front. Microbio. 1:137. doi: 10.3389/fmicb.2011.00137

Lin, M., Kikuchi, T., Brewer, H., M., Norbeck, A., D., and Rikihisa, Y. (2011). Global proteomic analysis of two tick-borne emerging zoonotic agents: Anaplasma phagocytophilum and Ehrlicia chaffeensis. Front. Microbio. 2:24. doi: 10.3389/fmicb.2011.00024

Ouellette, S., P. and Carabeo, R. A. (2010). A functional slow recycling pathway of transferring is required for growth of Chlamydia. Front. Microbio. 1:112. doi: 10.3389/fmicb.2010.00112

Stone, C., B., Bulir, D., C., Emdin, C., A., Pirie, R., M., Porfilio, E., A., Slootstra, J., W., and Mahony, J. B.
(2011). C. pneumoniae CdsL regulates CdsN ATPase activity, and disruption with a peptide mimetic prevents bacterial invasion. Front. Microbio. 2:21. doi: 10.3389/fmicb.2011.00021

Thompson, C., C. and Carabeo, R. A. (2011). An optimal method of iron starvation of the obligate intracellular pathogen, Chlamydia trachomatis. Front. Microbio 2:20. doi: $10.3389 /$ fmicb. 2011.00020

Wolf, K. (2011). Is C. pneumoniae research in peril? Front. Microbio. 2:56. doi: 10.3389/fmicb.2011.00056

Zhong, G. (2011). Chlamydia trachomatis secretion of proteases for manipulating host signaling pathways. Front. Microbio. 2:14. doi: 10.3389/ fmicb.2011.00014

Received: 19April 2011; accepted: 20 April 2011; published online: 05 May 2011.

Citation: Fields KA, Heinzen RA and Carabeo R (2011) The obligate intracellular lifestyle. Front. Microbio. 2:99. doi: 10.3389/fmicb.2011.00099

This article was submitted to Frontiers in Cellular and Infection Microbiology, a specialty of Frontiers in Microbiology.

Copyright (C) 2011 Fields, Heinzen and Carabeo. This is an open-access article subject to a non-exclusive license between the authors and Frontiers Media SA, which permits use, distribution and reproduction in other forums, provided the original authors and source are credited and other Frontiers conditions are complied with. 